\title{
ATRIBUCIONES CAUSALES EN LENGUAJE COMO PREDICTORAS DE RESPUESTAS DE ANSIEDAD
}

\author{
Aitana Fernández-Sogorb \\ Universidad de Alicante \\ aitana.fernandez@ua.es \\ María Vicent Juan \\ Universidad de Alicante \\ maria.vicent@ua.es \\ Carlos Edisson Jiménez Ayala \\ Universidad Central del Ecuador \\ cjimeneza@hotmail.com \\ Nancy Cargua García \\ Universidad Central del Ecuador \\ ncarguag@yahoo.es
}

Recepción Artículo: 22 octubre 2021

Admisión Evaluación: 22 octubre 2021

Informe Evaluador 1: 23 octubre 2021

Informe Evaluador 2: 24 octubre 2021

Aprobación Publicación: 24 octubre 2021

\section{RESUMEN}

La ansiedad escolar consiste en un conjunto de respuestas de tipo cognitivo, conductual y psicofisiológico que los estudiantes emiten cuando perciben situaciones del ámbito escolar como una amenaza. Aunque el alumnado puede tener mayor probabilidad de manifestar respuestas de ansiedad escolar según su estilo atribucional en el área de Lenguaje, la literatura científica existente sobre la relación entre ambos constructos en la adolescencia es limitada. El presente estudio se propuso examinar la capacidad predictiva de las atribuciones causales en Lenguaje sobre las tres respuestas de ansiedad escolar: ansiedad cognitiva, ansiedad conductual y ansiedad psicofisiológica. Se administró el Inventario de Ansiedad Escolar (IAES) y la Sydney Attribution Scale (SAS) a 586 estudiantes de entre 12 y 18 años $(M=14.82 ; D E=1.86)$. Los modelos de regresión logística revelaron valores de la odd ratio (OR) superiores a 1 para el fracaso en lenguaje atribuido al esfuerzo y a la capacidad en ansiedad cognitiva, ansiedad conductual y ansiedad psicofisiológica. Los valores de la OR para el resto de las atribuciones causales examinadas fueron inferiores a 1 en las tres respuestas de ansiedad escolar. Por tanto, se concluye que al aumentar las puntuaciones en atribuciones de éxitos y fracasos en lenguaje a causas externas disminuye la probabilidad de presentar alta ansiedad cognitiva, alta ansiedad conductual y alta ansiedad psicofisiológica. Sin embargo, a medida que aumentan las puntuaciones en las variables de atribuciones causales de los 


\section{ATRIBUCIONES CAUSALES EN LENGUAJE COMO PREDICTORAS DE RESPUESTAS DE ANSIEDAD}

fracasos a causas internas aumenta la probabilidad de presentar altos niveles en las tres respuestas de ansiedad. Estos hallazgos facilitarán el diseño de intervenciones dirigidas a cambiar el estilo atribucional de los adolescentes hacia atribuciones causales adaptativas.

Palabras clave: atribuciones causales; lenguaje; ansiedad escolar; adolescencia

\section{ABSTRACT}

Predictive capacity of attributional style in language on the manifestation of high levels of school anxiety responses. School anxiety consists of a set of cognitive, behavioral, and psychophysiological responses that students emit when they perceive situations in the school setting as a threat. Although students may be more likely to manifest school anxiety responses according to their attributional style in the area of language, the existing scientific literature on the relationship between both constructs in adolescence is limited. The present study aimed to examine the predictive capacity of causal attributions in language on the three school anxiety responses: cognitive anxiety, behavioral anxiety and psychophysiological anxiety. The School Anxiety Inventory (SAI) and the Sydney Attribution Scale (SAS) were administered to 586 students between 12 and 18 years old $(M=14.82 ; S D=1.86)$. Logistic regression models revealed odd ratio $(O R)$ values higher than 1 for failure in language attributed to effort and ability in cognitive anxiety, behavioral anxiety and psychophysiological anxiety. The OR values for the rest of the causal attributions examined were less than 1 in the three school anxiety responses. Therefore, it is concluded that by increasing the scores in attributions of successes and failures in language to external causes, the probability of presenting high cognitive anxiety, high behavioral anxiety, and high psychophysiological anxiety decreases. However, as the scores in the variables of causal attributions of failures to internal causes increase, the probability of presenting high levels in the three anxiety responses increases. These findings will facilitate the design of interventions aimed at changing the attributional style of adolescents towards adaptive causal attributions.

Keywords: causal attributions; language; school anxiety; adolescence

\section{INTRODUCCIÓN}

Altos niveles de ansiedad ante situaciones del ámbito educativo pueden conllevar el estrés e incluso el rechazo escolar (Fernández-Sogorb et al., 2018, 2021). La ansiedad escolar se manifiesta con respuestas de carácter cognitivo, conductual y psicofisiológico ante situaciones escolares que se perciben como un peligro (GarcíaFernández et al., 2011).

Cuando hablamos de autoatribuciones académicas nos referimos a un proceso en donde establecemos las causas de nuestros éxitos y fracasos. Esta teoría cognitiva elaborada por Weiner, como su principal exponente, señala que cuando un resultado es negativo, inesperado e importante, los estudiantes tienden a preguntarse por las causas que han originado tal resultado (Martinko y Mackey, 2019). Así, las causas que los estudiantes consideran como explicativas de sus resultados (atribución causal) favorecen la interpretación y valoración de estos resultados y determinan la motivación con la que el estudiante se enfrenta a una determinada situación académica.

El estudio de las atribuciones causales resulta interesante, el pensamiento atribucional se modifica en base a la evolución cognitiva del individuo (Gonzálvez et al., 2021). Almeida et al. (2008), en una muestra de 868 estudiantes portugueses de $5^{0}$ a $9^{0}$ grado destacaron que las chicas, en comparación con sus compañeros, atribuyeron sus éxitos en mayor medida al esfuerzo y al método de estudio, mientras los chicos consideraron que sus éxitos fueron debidos en mayor medida a la capacidad y a la suerte. No se encontraron diferencias respecto a los fracasos. Así, tanto chicos como chicas atribuyeron sus fracasos a la falta de esfuerzo o a la utilización de métodos de estudio inapropiados.

El estudio Ilevado a cabo recientemente por Inglés et al. (2011) en una muestra de 2.022 estudiantes de secundaria destacó que las diferencias de sexo en atribuciones causales se relacionaron con la materia de estudio (Matemáticas o Lengua). Así, los chicos, en comparación con sus compañeras, presentaron una mayor probabi- 
lidad de atribuir sus éxitos en matemáticas a la capacidad y sus fracasos a la falta de esfuerzo. Mientras, las chicas, presentaron una mayor probabilidad de atribuir sus éxitos en esta materia a su esfuerzo y los fracasos a su falta de capacidad. En relación con las tareas verbales, las chicas, en comparación con sus compañeros, presentaron una mayor probabilidad de atribuir sus éxitos a su capacidad y esfuerzo y sus fracasos a causas externas. Sin embargo, sus compañeros presentan una mayor probabilidad de atribuir sus fracasos en lengua a su capacidad.

\section{OBJETIVOS DE LA INVESTIGACIÓN}

Partiendo de la revisión de la literatura previa realizada, el presente estudio se propuso determinar la influencia del estilo atribucional en el área de lenguaje sobre respuestas de ansiedad escolar en adolescentes ecuatorianos. Las hipótesis planteadas fueron las siguientes: 1) se esperaba que conforme aumentaran las puntuaciones en atribuciones de éxito a la capacidad y al esfuerzo disminuyera la probabilidad de presentar alta ansiedad escoIar; 2) que conforme aumentaran las puntuaciones en atribuciones de fracaso a la capacidad y al esfuerzo aumentara la probabilidad de presentar alta ansiedad escolar; y 3) que conforme aumentaran las puntuaciones en atribuciones de éxito y fracaso a causas externas disminuyera la probabilidad de presentar alta ansiedad escolar.

\section{PARTICIPANTES}

586 adolescentes ecuatorianos de entre 12 y 18 años ( $M=14.82 ; D E=1.86)$ participaron en este estudio. Los nueve centros que participaron en el estudio se seleccionaron llevando a cabo un muestreo por conglomerados.

\section{MÉTODO}

\section{Instrumentos}

Inventario de Ansiedad Escolar (IAES; García-Fernández et al., 2011).

El IAES es un instrumento que evalúa el nivel de ansiedad en adolescentes de edades comprendidas entre los 12 y los 18 años. Su estructura está conformada por 25 ítems referidos a situaciones escolares, ubicadas en el eje horizontal de una tabla de doble entrada y 19 respuestas ( 9 de tipo cognitivas, 5 de tipo conductuales y 5 de tipo psicofisiológicas) ubicadas en el eje vertical de esta misma tabla. Para contestar el sujeto debe valorar la respuesta en función de la situación a partir de una escala de 5 puntos (0: nunca, 4: siempre).

Sydney Attribution Scale (SAS; Marsh, 1984).

Evalúa las percepciones de los sujetos sobre las causas de sus éxitos y fracasos académicos. Consta de 24 supuestos a los que los sujetos deben responder según una escala tipo Likert de cinco puntos (1: Falso/5: Verdadero).

\section{Procedimiento}

Tras llevar a cabo una reunión con el equipo directivo de cada centro, se solicitó el consentimiento a los padres de los participantes por escrito. La administración de los instrumentos a los adolescentes fue voluntaria y se preservó el anonimato.

\section{Análisis de datos}

La capacidad predictiva se halló con la regresión logística, mediante el estadístico de Wald. Se hizo uso de la odd ratio (OR), aplicando el criterio propuesto por De Maris (2003): si OR > 1, la predicción es positiva; si OR $<1$, la predicción es negativa, y si $O R=1$, no hay capacidad predictiva.

Para efectuar los análisis se usó del programa SPSS/PC 24.0. 


\section{RESULTADOS ALCANZADOS}

\section{Regresión logística binaria para la probabilidad de presentar alta ansiedad cognitiva en función de las atribuciones en lenguaje}

Los modelos resultantes (véase Tabla 1) permiten hacer una estimación correcta del $55.2 \%$ de los casos $\left(\chi^{2}\right.$ $=11.55 ; p=<.05)$ para la variable LEC = Éxito en el lenguaje atribuido a la capacidad; de un $56,4 \%$ de los casos $\left(\chi^{2}=18.18 ; p=<.05\right)$ para la variable de Atribución en LFEs= Fracaso en lenguaje atribuido al esfuerzo; de un $57,2 \%$ de los casos $\left(\chi^{2}=23.97 ; p=<.05\right)$ para la variable de Atribución en LEES= Éxito en lenguaje atribuido al esfuerzo; de un $57.6 \%$ de los casos $\left(\chi^{2}=26.62 ; p=<.05\right)$ correspondiente a la variable de Atribución en LFEX= Fracaso en lenguaje atribuido a causas externas y de un $59.6 \%$ de los $\operatorname{casos}\left(\chi^{2}=28.07 ; p=<.05\right)$ en la variable en LFC = Lenguaje Fracaso Capacidad. El valor de ajuste de los modelos ( $\mathrm{R}^{2}$ Nagelkerke) se situó entre 02 para el modelo de LEC=Éxito en lenguaje atribuido a la capacidad; y de .05 para el modelo Atribución de LFC = Lenguaje Fracaso Capacidad.

Los valores de la odd ratio $(O R)$ fueron superiores a 1 indicando, por tanto, que a medida que aumenta la puntuación en las variables de Atribuciones Causales en Lenguaje aumenta la posibilidad de presentar altas puntuaciones en ansiedad cognitiva, obteniendo valores para los modelos de LFES= Fracaso en lenguaje atribuido al esfuerzo de 1.56; y de 1.52 para LFC = Lenguaje Fracaso Capacidad, veces mayor por cada punto que aumentan las puntuaciones respectivamente en estas de atribuciones causales. A su vez, también se obtuvieron valores de la $O R$ inferiores a 1, por lo que la probabilidad de presentar alta ansiedad escolar para el Factor III fue de .74 en LEC=Éxito en lenguaje atribuido a la capacidad; en Atribución LEEx=Éxito en lenguaje atribuido a causas externas de .66; y de .58 en LFEX= Fracaso en lenguaje atribuido a causas externas, veces menor por cada punto que se incrementaban las puntuaciones en dichas variables.

Tabla 1

Regresión logística binaria para la probabilidad de presentar alta ansiedad cognitiva en función de las atribuciones en lenguaje

\begin{tabular}{|c|c|c|c|c|c|c|c|c|c|}
\hline $\begin{array}{l}\text { Variabl } \\
\mathrm{e}\end{array}$ & & $\chi^{2}$ & $\mathrm{R}^{2}$ & B & $\begin{array}{c}\text { E.T } \\
.\end{array}$ & Wald & $p$ & $O R$ & $\begin{array}{l}\text { I.C. } \\
95 \%\end{array}$ \\
\hline \multirow[t]{2}{*}{ LEC } & $\begin{array}{l}\text { Clasificados } \\
\text { correctament } \\
\text { e: } 55.2 \%\end{array}$ & $\begin{array}{r}11.5 \\
5\end{array}$ & $\begin{array}{r}.0 \\
2\end{array}$ & -.29 & $\begin{array}{r}.0 \\
8\end{array}$ & $\begin{array}{r}11.2 \\
5\end{array}$ & $\begin{array}{r}.00 \\
1\end{array}$ & .74 & $\begin{array}{r}.62- \\
.88\end{array}$ \\
\hline & Constante & & & $\begin{array}{r}1.0 \\
0\end{array}$ & $\begin{array}{r}.2 \\
6\end{array}$ & $\begin{array}{r}14.0 \\
0\end{array}$ & $\begin{array}{r}.00 \\
0\end{array}$ & 2.72 & \\
\hline \multirow[t]{2}{*}{ LEEx } & $\begin{array}{l}\text { Clasificados } \\
\text { correctament } \\
\text { e: } 57.2 \%\end{array}$ & 23.97 & .04 & -.41 & $\begin{array}{r}.0 \\
8\end{array}$ & $\begin{array}{r}22.9 \\
4\end{array}$ & $\begin{array}{r}.00 \\
0\end{array}$ & .66 & $\begin{array}{r}.55- \\
.88\end{array}$ \\
\hline & Constante & & & $\begin{array}{r}1.9 \\
5\end{array}$ & $\begin{array}{r}.3 \\
8\end{array}$ & $\begin{array}{r}25.5 \\
2\end{array}$ & $\begin{array}{r}.00 \\
0\end{array}$ & 7.09 & \\
\hline \multirow[t]{2}{*}{ LFC } & $\begin{array}{l}\text { Clasificados } \\
\text { correctament } \\
\text { e: } 59.6 \%\end{array}$ & 28.07 & $\begin{array}{r}.0 \\
5\end{array}$ & .42 & $\begin{array}{l}.0 \\
8\end{array}$ & $\begin{array}{r}26.8 \\
3\end{array}$ & $\begin{array}{r}.00 \\
0\end{array}$ & 1.52 & $\begin{array}{r}1.30 \\
- \\
1.79\end{array}$ \\
\hline & Constante & & & -.43 & $\begin{array}{r}.1 \\
3\end{array}$ & $\begin{array}{r}10.9 \\
7\end{array}$ & $\begin{array}{r}.00 \\
1\end{array}$ & .64 & \\
\hline \multirow[t]{2}{*}{ LFEs } & $\begin{array}{l}\text { Clasificados } \\
\text { correctament } \\
\text { e: } 56.4 \%\end{array}$ & 18.18 & .03 & .44 & $\begin{array}{l}.1 \\
0\end{array}$ & $\begin{array}{r}17.5 \\
8\end{array}$ & $\begin{array}{r}.00 \\
0\end{array}$ & 1.56 & $\begin{array}{r}1.26 \\
- \\
1.92\end{array}$ \\
\hline & Constante & & & -.74 & $\begin{array}{r}.2 \\
2\end{array}$ & $\begin{array}{r}11.2 \\
0\end{array}$ & $\begin{array}{r}.00 \\
1\end{array}$ & .47 & \\
\hline \multirow[t]{2}{*}{ LFEx } & $\begin{array}{l}\text { Clasificados } \\
\text { correctament } \\
\text { e: } 57.6 \%\end{array}$ & 26.62 & .04 & -.53 & $\begin{array}{l}.1 \\
0\end{array}$ & $\begin{array}{r}25.4 \\
4\end{array}$ & $\begin{array}{r}.00 \\
0\end{array}$ & .58 & $\begin{array}{r}.47- \\
.72\end{array}$ \\
\hline & Constante & & & $\begin{array}{r}2.4 \\
0\end{array}$ & $\begin{array}{r}.4 \\
5 \\
\end{array}$ & $\begin{array}{r}27.8 \\
4 \\
\end{array}$ & $\begin{array}{r}.00 \\
0\end{array}$ & $\begin{array}{r}11.0 \\
4\end{array}$ & \\
\hline
\end{tabular}

Nota LEC = Éxito en lenguaje atribuido a la capacidad; LEEx= Éxito en lenguaje atribuido a causas externas; $\mathrm{LFC}=$ Fracaso en lenguaje atribuido a la capacidad; $\mathrm{LFEs}=$ Fracaso en lenguaje atribuido al esfuerzo; $\mathrm{LFEx}=$ Fracaso en lenguaje atribuido a causas externas 


\section{Modelo de regresión logística para la probabilidad de presentar alta ansiedad cognitiva en fun- ción de las atribuciones en lenguaje incluyendo todas las variables}

La Tabla 2 presenta el modelo de regresión logística para Ansiedad Cognitiva, resultante tras incluir todas las variables de atribuciones causales en el área de Lenguaje. Los resultados muestran que la proporción de casos clasificados correctamente es del $65 \%\left(\chi^{2}=114.37 ; p=<.05\right)$. Los componentes del modelo expresados por la OR permiten pronosticar una alta ansiedad cognitiva, siendo 1.69 veces mayor la probabilidad de manifestar ansiedad escolar por cada punto que se incrementa la puntuación en Éxito en lenguaje atribuido al esfuerzo, y de 1.25 en Fracaso en Lenguaje atribuido a la Capacidad. Por otro lado, resultó ser .61, .80 y .66 veces menor la probabilidad de presentar alta ansiedad escolar por cada punto que aumentaba la puntuación en Éxito en Lenguaje atribuido a la Capacidad, Éxito en Lenguaje atribuido a causas Externas, y Fracaso en lenguaje atribuido a causas Externas.

Tabla 2

Modelo de regresión logística para la probabilidad de presentar alta ansiedad cognitiva en función de las atribuciones en lenguaje incluyendo todas las variables

\begin{tabular}{|c|c|c|c|c|c|c|c|c|c|}
\hline $\begin{array}{l}\text { Variabl } \\
\mathrm{e}\end{array}$ & & $\chi^{2}$ & $\mathrm{R}^{2}$ & B & E.T. & Wald & $P$ & $O R$ & $\begin{array}{r}\text { I.C. } \\
95 \%\end{array}$ \\
\hline & $\begin{array}{l}\text { Clasificados } \\
\text { correctamente } \\
: 61.6 \%\end{array}$ & $\begin{array}{r}67.7 \\
4\end{array}$ & $\begin{array}{l}.1 \\
1\end{array}$ & & & & & & \\
\hline LEC & & & & -.48 & $\begin{array}{l}.1 \\
3\end{array}$ & $\begin{array}{r}13.2 \\
2\end{array}$ & $\begin{array}{r}.00 \\
0\end{array}$ & .61 & $\begin{array}{r}.47- \\
.80\end{array}$ \\
\hline LEEs & & & & .52 & $\begin{array}{r}.1 \\
3\end{array}$ & $\begin{array}{r}15.6 \\
6\end{array}$ & $\begin{array}{r}.00 \\
0\end{array}$ & 1.69 & $\begin{array}{r}1.30 \\
- \\
2.19\end{array}$ \\
\hline LEEx & & & & -.22 & $\begin{array}{r}.1 \\
0\end{array}$ & 4.70 & $\begin{array}{r}.03 \\
0\end{array}$ & .80 & $\begin{array}{r}.65- \\
.98\end{array}$ \\
\hline LFC & & & & .22 & $\begin{array}{l}.0 \\
9\end{array}$ & 6.14 & $\begin{array}{r}.01 \\
3\end{array}$ & 1.25 & $\begin{array}{r}1.04 \\
- \\
1.49\end{array}$ \\
\hline LFEx & & & & -.40 & $\begin{array}{r}.1 \\
1\end{array}$ & $\begin{array}{r}11.9 \\
3\end{array}$ & $\begin{array}{r}.00 \\
1\end{array}$ & .66 & $\begin{array}{r}.52- \\
.83\end{array}$ \\
\hline & Constante & & & $\begin{array}{r}2.4 \\
5 \\
\end{array}$ & $\begin{array}{l}.6 \\
6\end{array}$ & $\begin{array}{r}13.7 \\
3\end{array}$ & $\begin{array}{r}.00 \\
0 \\
\end{array}$ & $\begin{array}{r}11.5 \\
8 \\
\end{array}$ & \\
\hline
\end{tabular}

Nota $:$ LEC= Éxito en lenguaje atribuido a la capacidad; LEEs= Éxito en lenguaje atribuido al esfuerzo; LEEx= Éxito en lenguaje atribuido a causas externas; LFC= Fracaso en lenguaje atribuido a la capacidad; $\mathrm{LFEx}=$ Fracaso en lenguaje atribuido a causas externas

\section{Regresión logística binaria para la probabilidad de presentar alta ansiedad conductual en fun- ción de las atribuciones en lenguaje}

Los modelos resultantes (véase la Tabla 3) permiten hacer una estimación correcta del $60.5 \%$ de los casos $\left(\chi^{2}=51.26 ; p=<.05\right)$ para la variable $L E C=$ Éxito en el lenguaje atribuido a la capacidad; de un $56.8 \%$ de los casos $\left(\chi^{2}=7.14 ; p=<.05\right)$ correspondiente a la variable de Atribución LEEs= Éxito en lenguaje atribuido al esfuerzo; de un $61.5 \%$ de los casos $\left(\chi^{2}=46.46 ; p=<.05\right)$ en la variable LEEX = Éxito en Lenguaje a Causas Externas; de un $59.8 \%$ de los casos $\left(\chi^{2}=26.9 ; p=<.05\right)$ en la Atribución LFC = Lenguaje Fracaso Capacidad; de un $57.1 \%$ de los casos $\left(\chi^{2}=9.07 ; p=<.05\right)$ en la Atribución LFEs= Fracaso en Lenguaje atribuido a la Falta de Esfuerzo y de un $58.5 \%$ de los casos $\left(\chi^{2}=28.10 ; p=<.05\right)$; para la variable de LFEX = Lenguaje Fracaso en Lenguaje a Causas Externas. El valor de ajuste de los modelos ( $R^{2}$ Nagelkerke) se situó entre .01 para el modelo de Éxito en lenguaje atribuido al esfuerzo; y de .09 para el modelo Atribución de Lenguaje Éxito Capacidad.

Los valores de la odd ratio $(O R)$ fueron superiores a 1 indicando, por tanto, que a medida que aumenta la puntuación en las variables de Atribuciones Causales en Lenguaje aumenta la posibilidad de presentar altas pun- 
tuaciones en ansiedad conductual, obteniendo valores para los modelos de Atribución del LFC = Lenguaje Fracaso Capacidad de 1.52; y de 1.36 en LFEs = Fracaso en Lenguaje atribuido a la Falta de Esfuerzo veces mayor por cada punto que aumentan las puntuaciones respectivamente en las dimensiones de atribuciones causales en estas variables. A su vez, también se obtuvieron valores de la $O R$ inferiores a 1, por lo que la probabilidad de presentar alta ansiedad conductual fue menor, concretamente de .78 en Éxito en Lenguaje atribuido al esfuerzo; en Atribución del Fracaso en Lenguaje a Causas Externas de .58; de .55 en Éxito en Lenguaje a Causas Externas; y de .51 en Éxito en lenguaje atribuido a la capacidad, por cada punto que se incrementaban las puntuaciones en

dichas variables.

Tabla 3

Regresión logística binaria para la probabilidad de presentar alta ansiedad conductual en función de las atribuciones en lenguaje

\begin{tabular}{|c|c|c|c|c|c|c|c|c|c|}
\hline $\begin{array}{l}\text { Variabl } \\
\text { e }\end{array}$ & & $\chi^{2}$ & $\mathrm{R}^{2}$ & B & E.T. & Wald & $P$ & $O R$ & $\begin{array}{l}\text { I.C. } \\
95 \%\end{array}$ \\
\hline \multirow[t]{2}{*}{ LEC } & $\begin{array}{l}\text { Clasificados } \\
\text { correctament } \\
\text { e: } 60.5 \%\end{array}$ & $\begin{array}{r}51.2 \\
6\end{array}$ & $\begin{array}{l}.0 \\
9\end{array}$ & -.67 & .10 & $\begin{array}{c}45 . \\
56\end{array}$ & $\begin{array}{r}.00 \\
0\end{array}$ & .51 & \multirow[t]{2}{*}{$.41-.62$} \\
\hline & Constante & & & 2.23 & .31 & $\begin{array}{r}51 . \\
17\end{array}$ & $\begin{array}{r}.00 \\
0\end{array}$ & 9.33 & \\
\hline \multirow[t]{2}{*}{ LEEs } & $\begin{array}{l}\text { Clasificados } \\
\text { correctament } \\
\text { e: } 56.8 \%\end{array}$ & 7.14 & $\begin{array}{c}.0 \\
1\end{array}$ & -.24 & .09 & $\begin{array}{r}6.9 \\
9\end{array}$ & $\begin{array}{r}.00 \\
8\end{array}$ & .78 & \multirow[t]{2}{*}{$.65-.93$} \\
\hline & Constante & & & .93 & .28 & $\begin{array}{c}11 . \\
00\end{array}$ & $\begin{array}{r}.00 \\
1\end{array}$ & 2.55 & \\
\hline \multirow[t]{2}{*}{ LEEx } & $\begin{array}{l}\text { Clasificados } \\
\text { correctament } \\
\text { e: } 61.5 \%\end{array}$ & 46.46 & .08 & -.59 & .09 & $\begin{array}{r}42 . \\
67\end{array}$ & $\begin{array}{r}.00 \\
0\end{array}$ & .55 & \multirow[t]{2}{*}{$.46-.65$} \\
\hline & Constante & & & 2.84 & .41 & $\begin{array}{r}47 . \\
80\end{array}$ & $\begin{array}{r}.00 \\
0\end{array}$ & $\begin{array}{r}17.2 \\
1\end{array}$ & \\
\hline \multirow[t]{2}{*}{ LFC } & $\begin{array}{l}\text { Clasificados } \\
\text { correctament } \\
\text { e: } 59.8 \%\end{array}$ & 26.09 & $\begin{array}{l}.0 \\
4\end{array}$ & .42 & .08 & $\begin{array}{r}24 . \\
89\end{array}$ & $\begin{array}{r}.00 \\
0\end{array}$ & 1.52 & \multirow[t]{2}{*}{$\begin{array}{r}1.29- \\
1.80\end{array}$} \\
\hline & Constante & & & -.34 & .13 & $\begin{array}{r}6.6 \\
3\end{array}$ & $\begin{array}{r}.01 \\
0\end{array}$ & .71 & \\
\hline \multirow[t]{2}{*}{ LFEs } & $\begin{array}{l}\text { Clasificados } \\
\text { correctament } \\
\text { e: } 57.1 \%\end{array}$ & 9.07 & .02 & .31 & .10 & $\begin{array}{r}8.9 \\
2\end{array}$ & $\begin{array}{r}.00 \\
3\end{array}$ & 1.36 & \multirow[t]{2}{*}{$\begin{array}{r}1.11- \\
1.67\end{array}$} \\
\hline & Constante & & & -.40 & .21 & $\begin{array}{r}3.3 \\
4\end{array}$ & $\begin{array}{r}.06 \\
7\end{array}$ & .67 & \\
\hline \multirow[t]{2}{*}{ LFEx } & $\begin{array}{l}\text { Clasificados } \\
\text { correctament } \\
\text { e: } 58.5 \%\end{array}$ & 28.10 & .05 & -.53 & .10 & $\begin{array}{r}26 . \\
79\end{array}$ & $\begin{array}{r}.00 \\
0\end{array}$ & .58 & \multirow[t]{2}{*}{$.47-.71$} \\
\hline & Constante & & & 2.49 & .44 & $\begin{array}{c}31 . \\
06\end{array}$ & $\begin{array}{r}.00 \\
0\end{array}$ & $\begin{array}{r}12.1 \\
0\end{array}$ & \\
\hline
\end{tabular}

Nota : LEC = Éxito en lenguaje atribuido a la capacidad; LEEs= Éxito en lenguaje atribuido al esfuerzo; LEEx= Éxito en lenguaje atribuido a causas externas; $L F C=$ Fracaso en lenguaje atribuido a la capacidad; LFEs= Fracaso en lenguaje atribuido al esfuerzo; $\mathrm{LFEx}=$ Fracaso en lenguaje atribuido a causas externas

\section{Modelo de regresión logística para la probabilidad de presentar alta ansiedad conductual en función de las atribuciones en lenguaje incluyendo todas las variables}

La Tabla 4 presenta el modelo de regresión logística para ansiedad conductual resultante tras incluir todas las variables de atribuciones causales en el área de Lenguaje. Los resultados muestran que la proporción de casos clasificados correctamente es del $64.1 \%\left(\chi^{2}=99.90 ; p=<.05\right)$. Los componentes del modelo expresados por la OR permiten pronosticar una alta ansiedad conductual, siendo 1.57 veces mayor la probabilidad de manifestar ansiedad escolar por cada punto que se incrementa la puntuación en Atribuciones LEEs= Éxito en lenguaje 
atribuido al esfuerzo. Por otro lado, resultó ser .42, .71 y .62 veces menor la probabilidad de presentar alta ansiedad escolar por cada punto que aumentaba la puntuación en Atribuciones del Éxito en Lenguaje a la Capacidad, Atribuciones de Éxito a causas Externas, y Atribuciones de Fracaso a causas Externas, respectivamente.

Tabla 4

Modelo de regresión logística para la probabilidad de presentar alta ansiedad conductual en función de las atribuciones en lenguaje incluyendo todas las variables

\begin{tabular}{|c|c|c|c|c|c|c|c|c|c|}
\hline $\begin{array}{l}\text { Variabl } \\
\mathrm{e}\end{array}$ & & $\chi^{2}$ & $\mathrm{R}^{2}$ & B & $\begin{array}{l}\text { E. } \\
\text { T. }\end{array}$ & Wald & $p$ & $O R$ & I.C. $95 \%$ \\
\hline & $\begin{array}{l}\text { Clasificad } \\
\text { os } \\
\text { correctame } \\
\text { nte: } 64.1 \%\end{array}$ & 99.90 & .16 & & & & & & \\
\hline LEC & & & & .86 & $\begin{array}{r}.1 \\
3\end{array}$ & $\begin{array}{r}38.8 \\
3\end{array}$ & $\begin{array}{r}.00 \\
0\end{array}$ & .42 & $.32-.55$ \\
\hline LEEs & & & & .45 & $\begin{array}{l}.1 \\
3\end{array}$ & $\begin{array}{r}11.1 \\
6\end{array}$ & $\begin{array}{r}.00 \\
1\end{array}$ & 1.57 & $\begin{array}{r}1.20- \\
2.05\end{array}$ \\
\hline LEEx & & & & .33 & $\begin{array}{r}.1 \\
0\end{array}$ & $\begin{array}{r}10.4 \\
6\end{array}$ & $\begin{array}{r}.00 \\
1\end{array}$ & .71 & $.58-.87$ \\
\hline LFEx & & & & .46 & $\begin{array}{r}.1 \\
1\end{array}$ & $\begin{array}{r}15.8 \\
7\end{array}$ & $\begin{array}{r}.00 \\
0\end{array}$ & .62 & .50 .79 \\
\hline & Constante & & & $\begin{array}{r}4.9 \\
5\end{array}$ & $\begin{array}{r}.6 \\
1\end{array}$ & $\begin{array}{r}64.9 \\
3 \\
\end{array}$ & $\begin{array}{r}.00 \\
0\end{array}$ & $\begin{array}{r}141 . \\
4\end{array}$ & \\
\hline
\end{tabular}

Nota: LEC $=$ Éxito en lenguaje atribuido a la capacidad; LEEs= Éxito en lenguaje atribuido al esfuerzo; LEEx= Éxito en lenguaje atribuido a causas externas; LFEx= Fracaso en lenguaje atribuido a causas externas

\section{Regresión logística binaria para la probabilidad de presentar alta ansiedad psicofisiológica en función de las atribuciones en lenguaje}

Los modelos resultantes (véase la Tabla 5) permiten hacer una estimación correcta del $58.8 \%$ de los casos $\left(\chi^{2}=24.07 ; p=<.05\right)$ para la variable LEC = Éxito en el lenguaje atribuido a la capacidad; de un $57.5 \%$ de los casos $\left(\chi^{2}=34.73 ; p=<.05\right)$ correspondiente a la variable de Atribución LEEX = Éxito en Lenguaje a Causas Externas; de un $58.9 \%$ de los casos $\left(\chi^{2}=25.54 ; p=<.05\right)$ en la variable LFC = Lenguaje Fracaso Capacidad; de un 55.1 de los casos $\left(5.16 \% \chi^{2}=25.54 ; p=<.05\right)$ en la Atribución LFEs: Fracaso en lenguaje atribuido al esfuerzo y de un $58.3 \%$ de los casos $\left(\chi^{2}=33.92 ; p=<.05\right)$; para la variable de LFEx = Lenguaje Fracaso en Lenguaje a Causas Externas. El valor de ajuste de los modelos ( $R^{2}$ Nagelkerke) se situó entre 01 para el modelo de Fracaso en lenguaje atribuido al esfuerzo; y de .06 para el modelo Éxito en Lenguaje a Causas Externas y Fracaso en Lenguaje a Causas Externas, respectivamente.

Los valores de la odd ratio $(O R)$ fueron superiores a 1 indicando, por tanto, que a medida que aumenta la puntuación en las variables de Atribuciones Causales en Lenguaje aumenta la posibilidad de presentar altas puntuaciones en ansiedad psicofisiológica, obteniendo valores para los modelos de Atribución de LFC = Lenguaje Fracaso Capacidad de 1.52; y en LFEs: Fracaso en lenguaje atribuido al esfuerzo de 1.27 veces mayor por cada punto que aumentan las puntuaciones respectivamente en las dimensiones de atribuciones causales en el área de Lenguaje indicadas. A su vez, también se obtuvieron valores de la OR inferiores a 1, por lo que la probabilidad de presentar alta ansiedad psicofisiológica fue de .63 en Éxito en el lenguaje atribuido a la capacidad; en Atribución Éxito en Lenguaje a Causas Externas de .60; y de .54 en Lenguaje Fracaso en Lenguaje a Causas Externas, veces menor por cada punto que se incrementaban las puntuaciones en dichas variables. 


\section{ATRIBUCIONES CAUSALES EN LENGUAJE COMO PREDICTORAS DE RESPUESTAS DE ANSIEDAD}

Tabla 5

Regresión logística binaria para la probabilidad de presentar alta ansiedad psicofisiológica en función de las atribuciones en lenguaje

\begin{tabular}{llcccccccc}
\hline Variable & $\chi^{2}$ & $\mathrm{R}^{2}$ & $\mathrm{~B}$ & $\mathrm{E} . \mathrm{T}$. & Wald & $P$ & OR & $\begin{array}{c}\text { I.C. } \\
95 \%\end{array}$ \\
\hline LEC & $\begin{array}{l}\text { Clasificados } \\
\text { correctamente: }\end{array}$ & 24.07 & .04 & -.44 & .09 & 22.65 & .000 & .63 & $.53-$ \\
& $\begin{array}{l}58.8 \% \\
\text { LEEx }\end{array}$ & & & 1.48 & .29 & 25.77 & .000 & 4.43 & .76 \\
& $\begin{array}{l}\text { Constante } \\
\text { Clasificados } \\
\text { correctamente: }\end{array}$ & 34.73 & .06 & -.50 & .08 & 32.61 & .000 & .60 & $.50-$ \\
& $\begin{array}{l}57.5 \% \\
\text { Constante }\end{array}$ & & & 2.36 & .39 & 35.52 & .000 & 10.66 & .71 \\
LFC & $\begin{array}{l}\text { Clasificados } \\
\text { correctamente: }\end{array}$ & 25.54 & .04 & .42 & .08 & 24.48 & .000 & 1.52 & $1.29-$ \\
& $\begin{array}{l}58.9 \% \\
\text { Constante }\end{array}$ & & & -.40 & .13 & 9.27 & .002 & .66 & 1.80 \\
LFEs & $\begin{array}{l}\text { Clasificados } \\
\text { correctamente: }\end{array}$ & 5.16 & .01 & .24 & .10 & 5.11 & .024 & 1.27 & $1.03-$ \\
& $\begin{array}{l}55.1 \% \\
\text { Constante }\end{array}$ & & & -.33 & .22 & 2.18 & .139 & .71 & 1.56 \\
LFEx & $\begin{array}{l}\text { Clasificados } \\
\text { correctamente: }\end{array}$ & 33.92 & .06 & -.60 & .10 & 32.00 & .000 & .54 & $.44-$ \\
& $\begin{array}{l}58.3 \% \\
\text { Constante }\end{array}$ & & & 2.69 & .45 & 34.72 & .000 & 14.77 & \\
\hline
\end{tabular}

Nota $:$ LEC $=$ Éxito en lenguaje atribuido a la capacidad; LEEx= Éxito en lenguaje atribuido a causas externas; $\mathrm{LFC}=$ Fracaso en lenguaje atribuido a la capacidad; $\mathrm{LFEs}=$ Fracaso en lenguaje atribuido al esfuerzo; $\mathrm{LFEx}=$ Fracaso en lenguaje atribuido a causas externas

Modelo de regresión logística para la probabilidad de presentar alta ansiedad psicofisiológica en función de las atribuciones en lenguaje incluyendo todas las variables

La Tabla 6 presenta el modelo de regresión logística resultante tras incluir todas las variables de atribuciones causales en el área de Lenguaje. Los resultados muestran que la proporción de casos clasificados correctamente es del $64.2 \%\left(\chi^{2}=88.29 ; p=<.05\right)$. Los componentes del modelo expresados por la OR permiten pronosticar una alta ansiedad psicofisiológica, siendo 2.01 veces mayor la probabilidad de manifestar ansiedad escolar por cada punto que se incrementa la puntuación en Atribuciones Éxito en lenguaje atribuido al esfuerzo. Por otro lado, resultó ser $.44, .74$ y .59 veces menor la probabilidad de presentar alta ansiedad escolar por cada punto que aumentaba la puntuación en Atribuciones del Lenguaje Éxito Capacidad, Atribuciones del Éxito en Lenguaje a causas Externas, Atribuciones de Fracaso en Lenguaje debido a causas Externas, respectivamente.

Tabla 6

Modelo de regresión logística para la probabilidad de presentar alta ansiedad psicofisiológica en función de las atribuciones en lenguaje incluyendo todas las variables

\begin{tabular}{|c|c|c|c|c|c|c|c|c|c|}
\hline Variable & & $\chi^{2}$ & $\mathrm{R}^{2}$ & B & E.T. & Wald & $p$ & $O R$ & $\begin{array}{l}\text { I.C. } \\
95 \% \\
\end{array}$ \\
\hline & $\begin{array}{l}\text { Clasificados } \\
\text { correctamente: } \\
64.2 \%\end{array}$ & 88.29 & .15 & & & & & & \\
\hline LEC & & & & -.80 & .14 & 32.74 & .000 & .44 & $.33-.58$ \\
\hline LEEs & & & & .69 & .14 & 23.53 & .000 & 2.01 & $\begin{array}{l}1.51- \\
2.66\end{array}$ \\
\hline LEEx & & & & -.29 & .10 & 8.37 & .004 & .74 & $.60-.90$ \\
\hline LFEx & & & & -.52 & .11 & 19.70 & .000 & .59 & $.46-.74$ \\
\hline & Constante & & & 4.04 & .59 & 46.52 & .000 & 57.00 & \\
\hline
\end{tabular}

Nota $:$ LEC $=$ Éxito en lenguaje atribuido a la capacidad; LEEs=Éxito en lenguaje atribuido al esfuerzo; LEEx= Éxito en lenguaje atribuido a causas externas; LFEx= Fracaso en lenguaje atribuido a causas externas 


\section{DISCUSIÓN}

En primer lugar, se examinó la probabilidad de presentar alta ansiedad cognitiva en función de las atribuciones causales en lenguaje. Los resultados obtenidos indican que a medida que aumenta la puntuación en las variables de atribuciones causales en lenguaje, aumenta la posibilidad de presentar altas puntuaciones en ansiedad cognitiva, obteniendo valores mayores para los modelos de fracaso en lenguaje atribuido al esfuerzo y a la capacidad. Este hallazgo permite confirmar la hipótesis 2 en lo que a la ansiedad cognitiva se refiere. Con respecto al modelo de regresión logística para la probabilidad de presentar alta ansiedad cognitiva en función de las atribuciones causales en lenguaje incluyendo todas las variables, los resultados permiten pronosticar una alta ansiedad cognitiva, siendo mayor la probabilidad de manifestar ansiedad escolar por cada punto que se incrementa el valor en éxito en lenguaje atribuido al esfuerzo y en fracaso en lenguaje atribuido a la capacidad.

Por otra parte, se identificó que a medida que aumenta la puntuación en las variables de atribuciones causales en lenguaje aumenta la posibilidad de presentar altas puntuaciones en ansiedad conductual, obteniendo valores mayores para los modelos de atribución del fracaso a la capacidad y a la falta de esfuerzo. A su vez, la probabilidad de presentar alta ansiedad conductual fue menor, concretamente en éxito en lenguaje atribuido al esfuerzo y a la capacidad, lo que permite confirmar la hipótesis 1.

Asimismo, los resultados obtenidos por los estudiantes indican que a medida que aumenta la puntuación en las variables de atribuciones causales en lenguaje aumenta la posibilidad de presentar altas puntuaciones en ansiedad psicofisiológica, obteniendo mayores valores para los modelos de atribución del fracaso a la capacidad y al esfuerzo, apoyando este hallazgo la segunda hipótesis del estudio. Los resultados también sustentan la tercera hipótesis, por la que se esperaba que conforme aumentaran las puntuaciones en atribuciones de éxito y fracaso a causas externas disminuyera la probabilidad de presentar altos niveles en las respuestas de ansiedad escolar. En definitiva, la capacidad predictiva del estilo atribucional en lenguaje de los adolescentes ecuatorianos sobre sus altos niveles en las respuestas de ansiedad escolar sigue la línea de la literatura previa (Almeida et al., 2008; Inglés et al., 2011).

\section{CONCLUSIONES}

Los resultados obtenidos en esta investigación permiten concluir principalmente que conforme aumentan las puntuaciones en atribuciones de éxito y fracaso atribuido a causas externas disminuye la probabilidad de presentar alta ansiedad escolar. No obstante, conviene que esas atribuciones sean realistas y controlables como el esfuerzo, por lo que la aplicación de programas de gestión emocional incluyendo un componente de reentrenamiento atribucional favorecería el desempeño académico y normal desarrollo de los adolescentes (FernándezSogorb et al., 2020).

\section{REFERENCIAS BIBLIOGRÁFICAS}

Almeida, L. D. S., Miranda, L. y Guisande, M. A. (2008). Atribuições causais para o sucesso e fracasso escolares. Estudos de Psicologia (Campinas), 25, 169-176.

Fernández-Sogorb, A., Inglés, C. J., Sanmartín, R., Gonzálvez, C., Vicent, M. y García-Fernández, J. M. (2018). Validation of the Visual Analogue Scale for Anxiety-Revised and school refusal across anxiety profiles. International Journal of Clinical and Health Psychology, 18(3), 264-272.

Fernández-Sogorb, A., Sanmartín, R., Vicent, M. y Gonzálvez, C. (2021). Identifying profiles of anxiety in late childhood and exploring their relationship with school-based distress. International Journal of Environmental Research and Public Health, 18(3), 948.

Fernández-Sogorb, A., Vicent, M., Gonzálvez, C., Sanmartín, R., Pérez-Sánchez, A. M. y García-Fernández, J. M. (2020). Attributional style in mathematics across anxiety profiles in spanish children. Sustainability, 12(3), 1173.

García-Fernández, J. M., Inglés, C. J., Martínez-Monteagudo, M. C., Marzo, J. C. y Estévez, E. (2011). Inventario 
de Ansiedad Escolar: validación en una muestra de estudiantes de Educación Secundaria. Psicothema, 23(2), 301-307.

Gonzálvez, C., Giménez-Miralles, M., Vicent, M., Sanmartín, R., Quiles, M. J. y García-Fernández, J. M. (2021). School Refusal Behaviour Profiles and Academic Self-Attributions in Language and Literature. Sustainability, 13(13), 7512.

Inglés, C. J., Díaz-Herrero, Á., García-Fernández, J. M. y Ruiz-Esteban, C. M. (2011). El género y el curso académico como predictores de las atribuciones en lectura y matemáticas en estudiantes de Educación Secundaria Obligatoria. Anales de Psicología, 27(2), 381-388.

Martinko, M. J. y Mackey, J. D. (2019). Attribution theory: An introduction to the special issue. Journal of Organizational Behavior, 40(5), 523-527.

Marsh, H. W. (1984). Relations among dimensions of self-attribution, dimensions of self-concept, and academic achievements. Journal of Educational Psychology, 76(6), 1291. 